\title{
The Spatial Correlation and Explanation of the Evolution of China's Regional Human Capital Structure-Based on Network Analysis Method
}

\author{
Xiao Dai ${ }^{1, *}$ and Liang Yan ${ }^{2}$ \\ 1 Business School, Hubei University, Wuhan 430062, China \\ 2 School of Economics and Management, China University of Geosciences, Wuhan 430074, China; \\ ylyzb@cug.edu.cn \\ * Correspondence: daixiao@hubu.edu.cn
}

Citation: Dai, X.; Yan, L. The Spatial Correlation and Explanation of the Evolution of China's Regional Human Capital Structure-Based on Network Analysis Method. Sustainability 2021, 13, 212.

https://doi.org/10.3390/su13010212

Received: 4 December 2020

Accepted: 24 December 2020

Published: 28 December 2020

Publisher's Note: MDPI stays neutral with regard to jurisdictional claims in published maps and institutional affiliations.

Copyright: () 2020 by the authors. Licensee MDPI, Basel, Switzerland. This article is an open access article distributed under the terms and conditions of the Creative Commons Attribution (CC BY) license (https: / / creativecommons.org/ licenses/by/4.0/).

\begin{abstract}
Human capital is an important catalyst for technology to play its role and the key to ensuring regional sustainable development. The current research on human capital mainly considers the imbalance of its stock and regional distribution without of the overall description of human capital structure and an analysis of its spatial correlation. Therefore, this article first builds a model of human capital structure evolution (HCSE) based on primary human capital, advanced human capital combined with economic geographic weight matrix, then uses the network analysis method to make a new deconstruction of the spatial correlation characteristics and the factors of China's regional HCSE from 2009 to 2018. The results show that: (1) China's HCSE has experienced regional accumulation in 2012, 2015, and 2018, having more prominent path dependence characteristics, which moreover vary significantly with China's provinces, that is, the eastern region is dominated by the path of outward overflow, and the central and western regions alternate inward input and outward output; (2) The spatial network of HCSE in China is stable and multi-superimposed. During the sample investigation period, the overall network density first increased and then decreased, the largest regional clustering coefficient and transmission efficiency appeared in 2014; (3) The eastern region (Beijing, Shanghai, and Guangdong as the core) is at the center of network spillover, playing a pivotal role as well as an intermediary and bridge role; conversely, the western region plays a central role in promoting the spatial network of HCSE to the direction of external dependence, while most of the central region has a diminished role in the evolution of spatial association networks; (4) The spatial correlation of HCSE in China is mainly affected by three types of factors: the urbanization level, the investment scale, and the degree of regional development. The greater the difference in urbanization levels is, the closer the spatial correlation of HCSE and the higher its network efficiency is; while the difference in investment scale and regional development has the opposite effect.
\end{abstract}

Keywords: human capital; spatial correlation; social network analysis

\section{Introduction}

The rapid development of China's economy in recent decades has benefited from the full use of China's regional comparative advantages in terms of manpower, geography, and industrial layout. The free flow of labor not only promotes the development of regional industries, but also narrows the economic gap between regions to a certain extent, especially the eastern coastal and western inland areas. Although these achievements are gratifying, there is no doubt that in the process of economic and urbanization development, the difficulty of obtaining resources, especially the increasing labor cost, has gradually weakened the original comparative advantage. The report of the 19th National Congress of the Communist Party of China pointed out that the "Chinese economy has shifted from a stage of rapid growth to a stage of high-quality development", the model of driving sustainable economic development in the world today has shifted from relying 
on natural resources to relying on technology. Many scholars have shown that human capital is the most important catalyst for technology [1-4] Meanwhile, Glaeser et al. (2014) further believe that human capital is the source of urban innovation and development [5]. The accumulation and concentration of human capital in cities is the foundation of urban economic development and an important source of modern economic growth [6].

With China's continuous investment in education, although the overall national education level has been continuously improving, there is still a large gap in the proportion of human capital compared with developed countries [7] (by 2014, the proportion of the population with a junior high school education and below has further dropped to $71.7 \%$, the proportion of the population with high school and higher education degrees increased to $16.1 \%$ and $12.2 \%$, respectively). At the same time, China has a vast territory, its regional development is complex and diverse, and the potential difference of economic and production factors have led to the flow of labor. Based on economic growth theory, the essence of population flow is the flow of human capital [8]. It is precisely because of the continuous flow of human capital that the development of human capital between provinces does not exist independently, but shows a certain spatial relationship [9]. Therefore, in order to improve the stock and quality of human capital, provinces and cities in China should consider not only their own industrial structure and development characteristics, but should also fully consider the external environment's attraction and spillover effect on human capital under the conditions of the spatial correlation of human capital. With the gradual formation and continuous implementation of China's four major economic sectors, the spatial connection of China's human capital becomes more extensive. These conditions make human capital development a road with a huge amount of space and a long way to go. However, the current research on human capital mainly focuses on its stock and distribution, while the research on the flow of human capital is mainly based on population mobility. This ignores changes in the nature of human capital; nor does it analyze the spatial correlation and complexity of human capital evolution. In this realistic situation, accurately portraying the overall network structure characteristics and the trend of the spatial correlation of the evolution of human capital structure closely related to regional development, clarifying the influence of each province and city on the development of the network structure, and further exploring the factors influencing the evolution of human capital structure all have important practical and theoretical significance for promoting the high-quality development of China's economy and improving labor output efficiency.

\section{Literature Review}

\subsection{Human Capital Structure Evolution (HCSE)}

To analyze human capital structural evolution, it is necessary to clarify the relevant research of human capital. For the study of human capital, foreign research can be traced back to Arrow's (1962) "learning by doing" theory. He believes that in the process of labor, the experience of the labor force continues to accumulate to promote the increase of overall productivity [10]. On this basis, Romer (1986) believes that an important source of economic growth is the need to understand human capital, technological innovation and knowledge diffusion [11]; Lucas (1999) used the "Two Economic Period Model" and the "Two Commodity Model" to study the factors affecting sustained economic growth and industrial development [12]. The results proved the key role of human capital accumulation and proposed that the continuous spillover of human capital must be maintained to promote the optimization of industrial structure.

At the same time, the increasingly complex economic structure and talent structure causes the coupling between human capital and real problems to deviate. Therefore, it is necessary to separate the homogeneity and heterogeneity of human capital. Academic circles have not reached a unified opinion on the definition or methods for measuring heterogeneous human capital, whether on the basis of either length of education or education level [13-15], or the educational Gini coefficient [16-20]. However, it is generally recognized that heterogeneous human capital is different from homogeneous human capi- 
tal, and it is nominally equivalent to concepts such as talented and high-quality human capital. The reason is that, in essence, talented and high-quality human capital are different from ordinary labor, and they are more able to bring irreplaceable creative profits to enterprises. In addition, they play a key role in technological innovation and absorptive capacity. From the perspective of changes in marginal returns, human capital will not decrease in the short term as a general labor force [21]. Given that human capital structure is closely related to the reform of the industrial structure and economic development, we believe that HCSE should be based on the ratio between homogeneous human capital and heterogeneous human capital.

In addition, HCSE also has typical path dependence characteristics.

From the perspective of human capital itself, its path dependence is significant. In recent years, the scope of application of path dependence has expanded to employment, career development and other fields. These practical experiences have attracted increasing attention towards the path dependence view for its revelatory capacity. It is generally believed that the accumulation of human capital occurs mainly through education and professional experience, so it is a long-term investment process for both individuals and organizations [22,23]. Therefore, human capital also means that labor mobility becomes more expensive [24]. Due to the large accumulation cost of human capital, its loss will have a greater negative impact on individuals than other equivalent benefits. With this awareness of continuous strengthening, human capital will soon form a path-dependent effect in time and space dimensions.

Some studies have shown that changes in human capital structure are better able to explain differences in regional development [13]. In the past few decades, many studies have used historical trajectories and events to explain the key leading factors of economic changes [25,26], basically covering the time and space dimensions. This view, emphasizing past influence, is typical high-level path dependence thinking, which can essentially help people understand the difference between system development and technological progress [27]. Based on the existing research results, we can easily construct a path dependence chain of HCSE from the perspective of regional development, that is, regional development has strong path dependence characteristics; meanwhile, changes in human capital structure are strongly related to regional development. Additionally, HCSE also has the nature of technological innovation. Therefore, the path dependence of HCSE must exist.

\subsection{The Spatial Correlation of Human Capital Structure Evolution}

First, government regulation and market mechanisms are important means for promoting the flow of production factors between regions. Looking at the development of China's economy, we can easily find that at different stages, the central government has launched some corresponding regional development strategies in a timely fshion based on the needs of different regions and the need to balance the country as a whole [28,29]. So far, four regional plate development blueprints have gradually been formed: "Western Development", "Northeast Revitalization", "Central Region Rise", and "Eastern First Development". The above policies aim to reduce the regional barriers to the flow of production factors across regions, so as to continuously strengthen the inter-regional linkages of production factors and reduce the development gap between regions [30,31]. In this process, as the human capital condensed in labor (as one production factor), its path development will inevitably form regional relationships. From a market perspective, with the continuous market-oriented reform of China's economy, the "invisible hand" of the market is naturally conducive to the free flow of production factors between provinces, municipalities, and regions, and it also promotes the spatial linkage of human capital [32,33].

Second, the disparity in development between regions makes the disparity in the stock and structural evolution direction of human capital increasingly obvious, which affects the spatial correlation of HCSE. For the Chinese labor market, one distinctive feature is the significant impact of a "diploma" on initial employment; in addition, the regional disparity in the rate of return of education. The superposition of these two effects directly affects 
the intention of human mobility [34]. On the other hand, compared with some foreign developed countries that pay more attention to the added value of labor "experience", Chinese companies pay more attention to the education level of human resources, and the uneven distribution of educational resources has brought about a regional imbalance in human capital structure. Therefore, when pursuing advanced human capital, enterprises tend to be in the regions with abundant and diversified human capital for the sake of cost and convenience, while the regions with relatively backward economies and education are ignored. Therefore, it is easier for regions to form a trajectory where the strong become the stronger and the weaker become even weaker. In view of this, this research believes that education and geographic elements must be combined with Chinese human capital structure evolution and its spatial correlation.

Finally, the disparity of HCSE has different spatial spillover effects. Enterprises and institutions in different regions have formed different spatial flows of human capital during economic activities. Additionally, driven by the scale effect, there has been a high regional accumulation of heterogeneous human capital and homogeneous human capital. Knowledge and innovation spillovers that promote sustainable economic development are closely related to regional human capital stock, which has triggered a new phenomenon. Namely, the impact of different human capital spillover effects on regional development is becoming more and more prominent. Luo Yong (2013) et al. compared the empirical results of heterogeneous human capital and homogeneous human capital in the free entrepreneur model of new economic geography, and found that the accumulation of heterogeneous human capital can shorten the cycle of reducing the level of regional specialization; meanwhile, when expanding the time for narrowing the income gap, homogeneous human capital has the opposite effect [14]. Chen Qiangyuan and Liang Qi (2014), Glaeser EL (2001), Anderson and S. Burges and Lane (2007) coupled heterogeneous cities from the perspective of heterogeneous labor and knowledge spillover, and integrated them into a framework of spatial economics, thus indirectly proving that the spatial spillover of human capital plays an important role in the scale development of cities and the urbanization of transition economies [35-37].

Therefore, it can be seen that the above studies have considered the stock and nature of human capital to varying degrees. Combining the relevant theories of economic geography and complexity science, we believe that HCSE involves more complex regional development models, geographical characteristics, technological innovation, educational foundation, etc., so HCSE have surpassed the purely geographical "near neighbor" relationship, and gradually formed a complex network structure with multiple feedback and dynamics.

So what is the current status of human capital in China? Can its degree and spatial correlation correspond to Chinese economic needs? The academic community cannot reach a unified opinion on this. Therefore, the possible innovation in this article are: (1) Analyze the connotation of HCSE and its characteristics in the development process; (2) Build an HCSE model with time-space effects by constructing the aggregation coefficient of homogeneous and heterogeneous human capital, then analyze the evolution characteristics of human capital structure in different regions; (3) Analyze the characteristics and complexity of the space correlation of HCSE, and deconstruct the main driving factors, thus providing a certain theoretical basis and policy guidance for formulating reasonable policies for Chinese HCSE in different regions.

\section{Research Ideas and Methods}

\subsection{The Measurement of HCSE}

As mentioned above, China's labor market has shifted from the prominent "demographic dividend" to the accumulation of human capital and "talent dividend". Therefore, the homogeneity assumption of human capital has caused an underestimation error in the estimation of human capital in industrial development and economic growth to a certain extent [14]. In view of this, scholars have also used different methods to re-estimate human 
capital in China. For example, Wang Yongshui (2018) decomposes human capital into B\&S-style innovation effects and imitation effects by adding a factor error structure to correct the effect on human capital growth [38]. Chen Xiaoguang (2005) takes into account the downward compatibility of human capital into the measurement [39]. The vector angle is one of the important methods to measure the employment structure of the labor force [40], especially for the measurement of structural changes in systems with multiple layers, the advantages are more obvious. Liu Zhiyong et al. (2018) carried out research on HCSE, constructing a hierarchical upgrading structure matrix of human capital [13]. According to the basic principle of the division of human capital in the previous article, HCSE is a process in which the population of high-education employment continues to grow, and the population of low-education and even illiterate and semi-illiterate practitioners declines. The specific construction process is as follows:

First, we divide human capital into homogeneous and heterogeneous human capital according to education level. This paper refers to Thomas et al. (2001) and Luo Yong et al. (2013) in order to calculate the aggregation coefficient of human capital by using the Gini coefficient $[3,14]$. Since the dataset is derived from different samples (basically classified by regions), the Gini coefficient of human capital is better able to indirectly reflect the spatial aggregation and distribution imbalance.

In accordance with the statistical classification of labor force education level in the China Labor Statistics Yearbook, we labeled the labor force that had never been to primary school, primary school, junior high school, high school, junior college, undergraduate, graduate student and above as $i(i=0,1, \cdots, 6)$, the corresponding number of employees as $n_{i}$, and the accumulated education years as $T_{i} ;$ then, we can make the following summary:

$$
T_{0}=0, T_{1}=6, T_{2}=9, T_{3}=12, T_{4}=15, T_{5}=16, T_{6}=15+\frac{3 n_{4}+4 n_{5}}{n_{4}+n_{5}}
$$

Then, the homogeneous labor is labeled as Labor $_{T}$, which is specifically expressed as:

$$
\text { Labor }_{T}=\left(n_{0}+n_{1}+n_{2}+n_{3}\right) *\left(n_{0}+\frac{n_{1} * n_{2}+n_{2} * n_{3}+2 n_{1} * n_{3}}{2 n_{1}+3 n_{2}+4 n_{3}}\right)
$$

while the heterogeneous labor is Labory:

$$
\text { Labor }_{Y}=\frac{\left(n_{4}+n_{5}+n_{6}\right) *\left(3 n_{5} n_{6}+4 n_{4} n_{6}+\frac{3 n_{4}+4 n_{5}}{n_{4}+n_{5}} n_{5} n_{4}+4 n_{5} n_{3}+3 n_{3} n_{4}\right)}{3 n_{4}+4 n_{5}+\left(3+\frac{3 n_{4}+4 n_{5}}{n_{4}+n_{5}}\right) * n_{6}}
$$

Then, according to Liu Zhiyong et al. (2018), the two types of human capital classified by education are regarded as one component of the space vector [13], thereby enabling the construction of a space vector, that is, $N_{0}=\left(n_{0,0}, n_{0,1}\right)$. Since the vector angle of each labor component is to be calculated, the reference vector must be set. The corresponding basic unit vector is $N_{0}^{\prime}$ :

$$
N_{0}^{\prime}=\left[\begin{array}{ccc}
n_{11} & \cdots & n_{1 j} \\
\vdots & \ddots & \vdots \\
n_{i 1} & \cdots & n_{i j}
\end{array}\right]=\left[\begin{array}{ccc}
1 & \cdots & 0 \\
\vdots & \ddots & \vdots \\
0 & \cdots & 1
\end{array}\right]
$$

Therefore, the angle $\theta_{j}$ is:

$$
\theta_{j}=\arccos \left\{\frac{\sum_{i=1}^{2}\left(n_{i, j}^{\prime} * n_{0, i}\right)}{\sqrt{\sum_{i=1}^{2} n \prime_{j, i}^{2}} * \sqrt{\sum_{i=0}^{2} n_{0, i}^{2}}}\right\}
$$

The weight of each angle is set to $W_{j}$, which is estimated on the basis of entropy estimation for the number of employees of different levels of labor, mainly due to the information dissipated during the operation of labor and industry systems. This is more 
consistent with the operating law of the system than simply defining it on the basis of years of education. Therefore, the level of human capital structure can be expressed as:

$$
\text { Labor }_{H}=\sum_{j=0}^{2}\left(W_{j} * \theta_{j}\right)
$$

So how is this variable measured? According to the design of industrial structure path-dependent, we define it as a variable strongly related to space and time. Therefore, two problems need to be solved: one is the calculation of the space matrix and the other is the basic path. This is because path dependence references past behavior rules due to the influence of bounded rationality and the relative closedness of the system during the process of institution or technology implementation. We accordingly label the basic path dependence as $L_{i t}$, which is used to compare the difference of the current and previous period to reflect the influence intensity of previous period. To reflect the importance of geographic space and the influence of regional development gaps, we adopt the method of Zhang et al. (2016), and take into account various calculation methods of spatial geographic weights [33]. Because the single definition of "one or zero" indicating "near or cut off" cannot represent the differences of industry and economy in different regions, we design the weight as a model based on the gravity model and label it as $w_{i j}$. Then, the following process can be observed:

$$
\begin{gathered}
\text { path }_{i t}=L_{i t}-\frac{1}{\sum_{j}^{m} w_{i j}} \sum_{j}^{m} w_{i j} * L_{j t} \\
L_{i t}=\frac{\text { Labor }_{i, t}-\text { Labor }_{i, t-1}}{\text { Labor }_{i, t-1}} \\
w_{i j}=\frac{K * G D P_{i} * G D P_{j}}{D_{i} * D_{j}} \\
\text { Labor }=\text { Labor }_{H}
\end{gathered}
$$

From the above formulas, it can be seen that the path-dependent construction sets up a spatial geographic weight matrix with regional economic conditions, mainly by introducing the gravity model, in which the initial index is the ratio of the current period to the previous period. Therefore, adding $L_{i t}$, path ${ }_{i t}$ produces the vector property on a mathematical level, that is, the comparison between directionality and size.

It should be noted that since the key variable "space" is embedded in path dependence, $w_{i j} * L_{j t}$ can be expressed as the relative overflow effect of human capital structure of the $j$ region and the $i$ region in the spatial dimension. In this way, we the extension of path dependence from a single time dimension to a "time + space" dimension. Therefore, we can roughly understand the path direction of HCSE in the $i$ region by observing the symbols of $p^{2} h_{i t}$. Specifically, if the value is positive, it indicates that the path direction mainly relies on the accumulation of its own human capital, which belongs to the regionalization of talent training, meaning that there is no obvious interaction between regions. However, the opposite indicates that the $i$ region has more connections with other regions in terms of advanced talents, making the effect of external human capital structure on that more comprehensive. Clearly, the change of the path ${ }_{i t}$ symbol shows the change of direction of HCSE.

\subsection{Spatial Correlation Characteristics and Complexity}

Through the above methods, we can roughly construct a HCSE model, and after substituting data, it is possible to obtain a basic understanding of the HCSE trend, especially during the inspection period, whether the path of each region is exported outward or absorbed inward. However, the disadvantage is that we cannot understand, in the context of spatial correlation, in which years Chinese HCSE and its characteristics of the spatial 
correlation is most obvious? Therefore, we need to use complexity science and related methods of social network analysis to do further research and exploration. Given that disordered time series contain more and more important information, the above information can only be obtained by projecting a given time series into a three-dimensional, four-dimensional or even higher-dimensional space. So there is still a question as to how to separate these subjects in the time domain to form a new sequence, namely so-called reconstructed phase spaces.

Therefore, this article is the first to use phase space reconstruction based on complexity science for obtaining the correlation dimension of HCSE at different times, finding several time points that changed significantly, followed by social network analysis.

\subsubsection{Phase Space Reconstruction and Phase Space Properties}

The original intention of reconstructing phase space was to try to find the chaotic attractor in the projected high-dimensional space, which is the key feature of the chaotic system (the laws of the chaotic system). The chaotic attractor is the stable orbit under the action of these laws, that is, the important information representing other components constitutes the chaotic attractor. The various disorder and chaotic phenomena of the original sequence in the time domain are caused by the chaotic attractor orbit after various stretching, compression, translation, and rotation of time and space. Through a large number of mathematical proofs, Takens (1981) gave the relationship between the embedding dimension $m$ and the motive power system dimension $d$ in 1981, namely $m \geq 2 d+1$, which indicates that when the phase space is reconstructed, if this relationship is satisfied, then in the constructed $R^{m}$ space, the chaotic attractor can be recovered well, and the two dynamic systems maintain the differential homology [41].

(1) Determine the delay time independently $[42,43]$

The determination of delay time determines the degree of correlation between components after reconstructing the phase space. If the delay time $\tau$ is too small, then the information contained in each component is mostly the same, which loses the meaning of reconstruction; if the delay time $\tau$ is too large, which makes the components independent of each other, the chaotic attractor trajectory obtained cannot summarize the laws of other components, so the appropriate delay time $\tau$ is of great significance. The autocorrelation method is a widely used and mature method to find the delay time $\tau$, and the judgment standard is based on the investigation of time series correlation. For continuous variables $x(t)$, the autocorrelation function $C(\tau)$ is defined as:

$$
C(\tau)=\lim _{T \rightarrow \infty} \frac{1}{T} \int_{-\frac{T}{2}}^{\frac{T}{2}} x(t) x(t+\tau) d t
$$

According to some experimental data results, the iteration loop does not end until the value of the autocorrelation function drops to $1-1 / 2.71828$ of the initial value, and the corresponding $\tau$ is the required delay time.

The definition of the autocorrelation function illustrates the degree of correlation between the value obtained after the time delay $\tau$ and the initial value. The larger the $C(\tau)$ is, the closer the $x(t)$ and $x(t+\tau)$ are. Similarly, the smaller the $\tau$ is, the larger $C(\tau)$ is. The former is obtained from the perspective of calculation, and the latter is obtained from the perspective of properties. Furthermore, since $C(\tau)$ is a standard even function, through a series of $C(\tau)$, the even oddness can also be investigated after reconstructing the phase space; at the same time, if $x(t)$ is a periodic function, then $C(\tau)$ is also periodic, which can reduce the workload. 
The above method applies for the continuous variable $x(t)$; for the time series $x_{1}, x_{2}, \cdots, x_{n}, \cdots$, the time delay of sequence $\left\{x_{i}\right\}$ is $j \tau$, and therefore the autocorrelation function can be defined as

$$
C R_{x x}(j \tau)=\frac{1}{N} \sum_{i=0}^{N-1} x_{i} x_{j \tau+i}
$$

\section{(2) Phase space properties}

After completing phase space reconstruction, we can determine whether a system has fractal or chaotic properties by calculating some invariants. Generally, the invariants often include correlation dimension D, Kolmogorov Entropy, and Lyapunov Exponent, etc. Based on the foregoing, we determined the delay time of phase space reconstruction, and the GP method not only determines the correlation dimension $D$, but also calculates the embedding dimension. Therefore, this paper adopts the GP method.

Regarding the mathematical meaning of system dimension from integer dimension to fractal dimension, Hausdorff first proposed a new definition in 1919, namely Harsdorff dimension [44], which expressed the system dimension as:

$$
C D_{0}=\lim _{r \rightarrow 0} \frac{\ln N(r)}{\ln \frac{1}{r}}
$$

The primary characteristic of the Harsdorff dimension is that it is topological dimension that is greater than Euclidean geometry and lesser than fractal dimension. Although the Harsdorff dimension cannot accurately describe the calculation of fractal dimension, it completes the preliminary mathematical definition. The information dimension, as an extended form of the Harsdorff dimension, makes a reasonable explanation from the perspective of information, laying the foundation for the development of information entropy. Information dimension based on probability, the correlation dimension $D$ can be expressed as

$$
D_{1}=\lim _{r \rightarrow 0} \frac{-\sum_{i=1}^{N(r)} p_{i} \ln p_{i}}{\ln \frac{1}{r}}
$$

In 1983, Grossberger and Procaccia proposed the concept of correlation integral and the corresponding mathematical formula based on the coordinate delay method, called the G-P algorithm [45]. Since then, the G-P method has been widely used in the calculation of correlation dimensions.

Assuming that the phase space has been reconstructed and the embedding dimension $m$ is determined, first, take a first total of $m$ data to form a sequence $\left\{x_{1}, x_{2}, \cdots, x_{m}\right\}$, the first point $r_{1}$ in the $m$ dimensional space can be determined, and then remove $x_{1}$, add $x_{m+1}$ to form a new sequence including $m$ data, thus determine the second point $r_{2}$. By analogy, a large number of phase points in $m$-dimensional space can be constructed, that are connected to form the trajectory in the line space. Then, the correlation of the phase points in this trajectory to get the dimension were examined. Supposing that the time series $\left\{x_{i}\right\}$ generates a total of $N$ phase points in the $m$-dimensional space, namely $r_{1}, r_{2}, \cdots, r_{i}, \cdots$, a number $r$ is given to traverse the distance between all phase points $\left\|r_{i}-r_{j}\right\|$, and to count how many pairs of points $\left(r_{i}, r_{j}\right)$ have a distance less than $r$,

$$
C(r)=\frac{1}{N} \sum_{i, j=1, i \neq j}^{N} \theta\left(r-\left\|r_{i}-r_{j}\right\|\right)
$$

where $\theta$ is the Heaviside step function, which is defined as

$$
H(x)=\left\{\begin{array}{l}
1 x \geq 0 \\
0 x \leq 0
\end{array}\right.
$$


The value of $r$ affects the validity of the final calculation result. The reason is that if $r$ is too large, it may cause the distance of all points to be less than it, then $C(r)=1, \ln C(R)=0$, so effective information cannot be extracted; while when $\mathrm{r}$ is reduced, there may be $r$, which makes $C(r) \rightarrow r^{D}$. If the above relationship can be realized, there is a correlation dimension $D_{2}$ :

$$
D_{2}=\lim _{r \rightarrow 0} \frac{\ln C(r)}{\ln r}
$$

when $r \rightarrow 0$, then there is

$$
\lim _{r \rightarrow 0} C(r)=\lim _{N \rightarrow \infty} \frac{1}{N(N-1)} \sum_{i=1, i \neq j}^{N} \sum_{j=1}^{N} \theta\left(r-\left\|r_{i}-r_{j}\right\|\right)
$$

\subsubsection{Network Structure}

Social network analysis is an important research method in new economic sociology [46]. Its core point is that if there is an interactive relationship between two members, then this relationship is important and complex. The research methods mainly use graph theory tools and algebraic model technology to describe relational patterns, and explore the influence of these relational patterns on the members and systems in the structure [47]. Therefore, social network analysis mainly focuses on the collection of actors and their relationships. Its special and effective feature makes it multi-faceted; in short, it studies the phenomena and structures in reality from the perspective of "relationships".

(1) Network density

If the overall network is an undirected network of relations, in which there are $n$ actors, then the theoretical maximum possible value of the total number of relations is $\frac{n(n-1)}{2}$. If the actual number of relationships contained in the network is $m$, then the network density is the "total number of relationships that actually exist" divided by the "total number of theoretically most possible relationships", that is, $\frac{m}{\frac{n(n-1)}{2}}=2 m /(n(n-1)$. If the overall network is a directed relationship network and there are $n$ actors in it, then the theoretical maximum possible value of the total number of relationships contained in it is $n(n-1)$, then the density of the network is $m /(n(n-1)$. Obviously, the greater the network density is, the closer the relationship between the members is.

(2) Network centrality [48,49]

Network centrality analysis is mainly divided into "point centrality" and "graph centrality". The former describes the power of individual members, while the latter describes the overall concentration or consistency of system.

The formula for calculating the graph central potential is:

$$
C=\frac{\sum_{i=1}^{n}\left(C_{\max }-C_{i}\right)}{\max \left[\sum_{i=1}^{n}\left(C_{\max }-C_{i}\right)\right]}
$$

Among them, $C_{\max }$ is the maximum centrality in the figure, and $C_{i}$ is the centrality of each point.

Furthermore, the point centrality can also be subdivided into: degree centrality, close centrality, and intermediate centrality. Degree centrality measures the degree to which a node is at the center of network, and its formula is:

$$
C_{D}\left(n_{i}\right)=\sum_{j=1}^{n} X_{j i}
$$

where $C_{D}\left(n_{i}\right)$ is the degree centrality, and $X_{j i}$ is the connection strength among nodes.

Proximity centrality measures the closeness of a node to all other nodes. Scholars such as Freeman regard it as the distance between points. Then it can be known that when 
a node's closeness centrality is high, it will be close to other nodes in the network. The formula is:

$$
C_{A P i}^{-1}=\sum_{j=1}^{n} d_{i j}
$$

where $C_{A P i}^{-1}$ is the closeness centrality of a node, and $d_{i j}$ is the shortcut distance between the nodes (that is, the number of shortcut distance).

The intermediate centrality measures the degree of node control over resources, and it acts as an intermediary to contact other nodes. This concept is more widely used in structural hole theory. The formula is:

$$
\begin{gathered}
C_{A B i}^{-1}=\sum_{j=1}^{n} \sum_{k}^{n} b_{i k}(i), i \neq j \neq k \\
b_{i k}(i)=g_{j k}(i) / g_{j k}
\end{gathered}
$$

where $C_{A B i}^{-1}$ is the intermediate centrality of node, and $j<k, b_{i k}(i)$ represents the third point $i$ controlling the ability of point $j$ and $k$ to communicate, that is, the probability that point $i$ is on the shortcut of nodes $j$ and $k$.

(3) Small world network

Before the small-world network was proposed, most studies believed that most networks belonged to the sparse adjacent coupling network and random network, but these two networks have stricter requirements on the node connections. The former has a larger characteristic path length and clustering coefficient, while the latter is just the opposite. Obviously, the above two networks are not enough to fit the real-world situation. Therefore, Watts and Strogtz proposed the small world network in 1998 [50]. They believed that the small world network exists widely in nature, society, physics and mathematics. Most nodes in the small world network are not connected to each other directly, but can build associative relationships through other points. Strictly speaking, a small world network is a network model with a shorter average path length and a larger clustering coefficient than a random network. In this way, a dynamic system with small-world characteristics can finally promote global changes through nodes' local behavior. While the relationship between local members and their dynamic behavior and the global network mainly depend on the network structure. Then, by comparing the small-world network characteristic indicators of different networks, namely the average path length and the clustering coefficient, the information propagation speed between networks can be clarified, that is, the smaller the average path length is, the higher the efficiency of the network is; and the larger the clustering coefficient is, the closer the connection between the network nodes is. The average path length $L$ of entire network is:

$$
L=\frac{1}{n(n-1)} \sum_{i \neq j} d_{i j}
$$

The network average clustering coefficient $C$ is:

$$
\begin{gathered}
C=\frac{1}{N} \sum_{i} c_{i} \\
c_{i}=\frac{1}{k_{i}\left(k_{i}-1\right)} \sum_{j \neq k} a_{i j} a_{i k} a_{j k}
\end{gathered}
$$

where $c_{i}$ is the clustering coefficient of node $i$, and $a_{i j}, a_{i k}, a_{j k}$ are the connections between node $i$ and its adjacent nodes. 


\section{Results Analysis}

\subsection{The Characteristics of HCSE}

\subsubsection{Basic Features}

As results shown in Appendix A, in most statistical regions, the volatility of HCSE is a low-amplitude fluctuation pattern. However, from 2009 to 2012 and 2014 to 2015, most regions underwent a major change process, indicating that human capital structures at this stage were going through adjustments. From a numerical perspective, HCSE in the eastern region was mostly greater than 0 , and the central and western regions were bounded by " 0 ", and the number of regions greater than " 0 " and less than " 0 " accounted for half. This shows that HCSE in the eastern region was mainly based on outward output, while the central and western regions currently have both inward input and outward output; however, the trend of inward input is more obvious. In other words, HCSE in the east has formed a certain spillover effect, while the central and western regions rely on external introductions without forming an effective adaptive effect of HCSE. According to the upgrading process of human capital structure, it is bound to be closely related to the change of industrial structure and economic development. Therefore, the HCSE trend can also reflect the development of different regions and different periods to a certain extent. From this perspective, human capital structure in the eastern region has formed a certain adaptive system with social and economic development, which can have a good radiation effect on the surrounding areas and further expand the social and economic development influence. Since the central and western regions are still mainly import-oriented, this shows that these two regions hope to promote a sustainable development of society and economy to break the limitations of current development through HCSE. However, because HCSE has not formed a good positive feedback with their respective systems, the spillover effect of HCSE cannot be come into being. Therefore, under the background of Chinese "new normal" and the practical requirements of industrial structure transformation, the path of HCSE faces more complicated interference, and its development process has a long way to go.

\subsubsection{Phase Space Properties}

In Table 1, we obtained the development trend and corresponding characteristics of Chinese HCSE in various regions from 2008 to 2018, pointing out the years with large fluctuations. To effectively clarify the spatial network structure characteristics and the complexity of HCSE, we need to further select the most influential years for specific indepth analysis. Therefore, this article draws on the relevant theories and methods of complexity science, for it can strip the redundant information in time series through phase space reconstruction to get the most critical impact year.

Table 1. Phase space reconstruction.

\begin{tabular}{cccccccccccc}
\hline Time & $\mathbf{2 0 0 8}$ & $\mathbf{2 0 0 9}$ & $\mathbf{2 0 1 0}$ & $\mathbf{2 0 1 1}$ & $\mathbf{2 0 1 2}$ & $\mathbf{2 0 1 3}$ & $\mathbf{2 0 1 4}$ & $\mathbf{2 0 1 5}$ & $\mathbf{2 0 1 6}$ & $\mathbf{2 0 1 7}$ & $\mathbf{2 0 1 8}$ \\
\hline Delay Time & 2 & 2 & 3 & 2 & 2 & 2 & 3 & 2 & 2 & 2 & 2 \\
Embedding Dimension & 2 & 2 & 2 & 2 & 2 & 2 & 2 & 2 & 2 & 2 & 2 \\
Correlation Dimension & 2.158 & 2.048 & 2.302 & 2.254 & 0.979 & 1.646 & 1.174 & 0.396 & 1.494 & 1.975 & 0.531 \\
\hline
\end{tabular}

The results show that, overall, the delay time and embedding dimension of the reconstructed phase space from 2008 to 2018 are the same in other years except for 2010 and 2014. However, on the contrary, the correlation dimension fluctuates greatly from 2008 to 2018. Specifically, 2008 2011 is a gradual rise stage reaching the peak in 2011, then falling rapidly in 2012, then slowly rebounding from 2012 to 2013, and after a sharp decline in 2015, it rises again to a peak in 2017. Since HCSE, as constructed in this article, has both spatial effects, the correlation dimension has the characteristics of fractal geometry, which can reflect the spatial equilibrium or agglomeration characteristics, that is, the larger the correlation dimension is, the more balanced spatial distribution of elements is, and 
on the contrary, the more concentrated in a certain place. From this, we can know that in 2008 2011, 2013 2014, 2016 2017, HCSE in China was relatively balanced, while regional agglomeration appeared in other years. At the same time, it can be seen that during the entire inspection period, there were turning points in 2011, 2014 and 2017. Therefore, we believe that these three years can be extracted to make a detailed analysis of the spatial correlation of HCSE.

\subsection{Spatial Correlation Characteristics}

\subsubsection{Network Density}

The higher the network density is, the closer and more frequent the connections between nodes are. As it is shown in Table 2, the network density of HCSE among provinces in China increases first and then decreases, indicating that the exchanges between provinces are relatively sufficient, which can promote the active flow of factors and further promote the balanced development of regions, which is similar to the result of correlation dimension. In addition, in 2014, the network density between provinces was the highest, either reflecting the increased diffusion capacity of HCSE among provinces or highlighting the advantages of factor agglomeration and the enhancement of scale effects. However, a higher network density does not necessarily bring sustainable positive feedback to a system, for it is mainly restricted by the balance of costs and benefits. Therefore, seeking an appropriate network scale and density can contribute to regional coordinated development.

Table 2. Network density.

\begin{tabular}{cccc}
\hline Time & $\mathbf{2 0 1 1}$ & $\mathbf{2 0 1 4}$ & $\mathbf{2 0 1 7}$ \\
\hline Network density & -0.009 & 0.007 & 0.001 \\
\hline
\end{tabular}

The above problems are closely related to the overall efficiency of network, so we need to understand the minimum distance required for network information transmission. Therefore, this research further conducts small-world analysis.

\subsubsection{Small World Network}

On the basis of the results in Table 3, it can be seen that the small world characteristics of the spatial network structure of HCSE are more obvious in 2014 than in 2011 and 2017, that is, it has smaller average path distances and larger clustering coefficient in 2014.

Table 3. Small world network.

\begin{tabular}{cccc}
\hline Time & $\mathbf{2 0 1 1}$ & $\mathbf{2 0 1 4}$ & $\mathbf{2 0 1 7}$ \\
\hline Average distance & 1.566 & 1.519 & 1.561 \\
Distance-based cohesion ("Compactness") & 0.728 & 0.749 & 0.742 \\
Distance-weighted fragmentation ("Breadth”) & 0.272 & 0.251 & 0.258 \\
Overall graph clustering coefficient & 0.589 & 0.635 & 0.643 \\
\hline
\end{tabular}

The average path lengths in 2011 and 2017 were similar, and they were both higher than in 2014. This indicates that the shortest path required for urban connections was in 2014, which means that the barriers to the spatial flow of human capital are low, and human capital in various regions can form complementary advantages, thus promoting regional coordinated development. The clustering coefficient shows that the slow upward trends, especially in 2014 and 2017, were not much different. This means that HCSE in 2014 and 2017 formed an agglomeration phenomenon, further intensifying the path development of HCSE in different regions. For regions with spillover effects, the small-world feature is obviously conducive to driving the development of surrounding areas and reducing redundant structures; on the contrary, for regions that are imported inward, the obvious small-world characteristics further strengthen the external dependence of HCSE, fail to 
promote those regions to break the existing development mode. We suppose that, to a certain extent, the investment in economic development is wasted and it is difficult to stimulate the dividend effect of human capital.

\subsubsection{Network Centrality}

First, on the basis of the results showed in the Table 4 and Appendix A, on the whole, the degree centrality, proximity centrality and intermediary centrality of the eastern region in 2011 were relatively high; in 2014, the degree centrality of the west was the highest, and the proximity centrality of the east, west, and central was not much different, and the intermediary centrality was still dominated by the east. The situation in 2017 and 2011 was similar, but the western region also showed certain advantages in the degree centrality and the intermediary centrality. This shows that, in most cases, the eastern region is at the core of HCSE, which not only reflects in its own control of resources (high degree centrality) and the ability to communicate with other regions (high closeness centrality), but also reflects in the role of connecting other nodes. Therefore, the eastern region plays a key role in the spatial network for Chinese HCSE. In addition, in 2014 and 2017, the degree centrality of the western region was also relatively high, indicating that the network density of HCSE in the western region has increased to a certain extent in 2014 compared with previous years, and its intersection with the central and eastern regions increasing. However, it is worth noting that in most years, HCSE in the western region is mainly based on external forces. Therefore, although the network centrality of the western region is better in 2014, its development is still too dependent on the outside world to form effective sustainable development. Similarly, although the intermediary centrality in the western region was relatively high in 2017, due to its development path characteristics, HCSE actually has many redundant relationships, that is, there is a waste of resources in development.

Table 4. Network centrality.

\begin{tabular}{|c|c|c|c|c|c|c|c|c|c|}
\hline \multirow{2}{*}{ Time } & \multicolumn{3}{|c|}{2011} & \multicolumn{3}{|c|}{2014} & \multicolumn{3}{|c|}{2017} \\
\hline & Degree & Closeness & Betweenness & Degree & Closeness & Betweenness & Degree & Closeness & Betweenness \\
\hline \multirow[t]{3}{*}{ The East } & Beijing & Beijing & Beijing & Shanghai & Shanghai & Shanghai & Guangdong & Guangdong & Guangdong \\
\hline & Guangdong & Guangdong & Guangdong & Zhejiang & Zhejiang & Jiangsu & Shandong & Shanghai & Shanghai \\
\hline & Hebei & Hebei & Hebei & Fujian & Fujian & Shandong & Shanghai & Hebei & Hebei \\
\hline \multirow{3}{*}{$\begin{array}{l}\text { The } \\
\text { Central }\end{array}$} & Jiangxi & Henan & Henan & Anhui & Anhui & Anhui & Hunan & Hunan & Hunan \\
\hline & Henan & Jiangxi & Jiangxi & Henan & Henan & Henan & Hubei & Neimenggu & Neimenggu \\
\hline & Hunan & Hunan & Hunan & Shanxi & Jiangxi & Shanxi & Neimenggu & Hubei & Anhui \\
\hline \multirow[t]{3}{*}{ The West } & Chongqing & Chongqing & Guizhou & Guizhou & Guizhou & Guizhou & Chongqing & Shanxi & Sichuan \\
\hline & Guangxi & Guizhou & Ningxia & Yunnan & Yunnan & Ningxia & Shanxi & Sichuan & Shanxi \\
\hline & Guizhou & Qinghai & Chongqing & Ningxia & Ningxia & Yunnan & Sichuan & Guizhou & Guizhou \\
\hline $\begin{array}{l}\text { The } \\
\text { Northest }\end{array}$ & Jilin & Jilin & Liaoning & Jilin & Jilin & Heilongjiang & Liaoning & Jilin & Heilongjiang \\
\hline $\begin{array}{l}\text { Network } \\
\text { centrality }\end{array}$ & & $6.426 \%$ & & & $5.227 \%$ & & & $8.318 \%$ & \\
\hline
\end{tabular}

Secondly, judging from the scope of the eastern, central, western regions, it can be clearly found that the three major nodes in the eastern region are Beijing, Shanghai, and Guangdong, exactly reflecting the core members of the "Beijing-Tianjin-Hebei", "Yangtze River Delta" and "Pearl River Delta" economic circles. The central region is dominated by Hunan and Henan; the western region is dominated by Guizhou, Chongqing, and Shaanxi. Combining the path direction of their respective HCSE, it can be seen that Beijing, Shanghai, and Guangdong can form an effective collection of human capital resources, which can not only exert the cluster spillover effect of HCSE, but also effectively build a bridge for the development of human capital in different regions, so that the surrounding human capital can flow effectively. Since HCSE of Hunan and Henan province in 2011 and 2014 was inward input, and 2017 was outward output, they therefore have a restraining effect on the spatial network of HCSE, as a result of strengthening the external dependence of their own development. This situation has been improved to a certain extent in 2017. 
Similarly, comparing Guizhou, Chongqing, and Shaanxi, we find that Shaanxi's positive effects occurred in 2011 and 2014, while Guizhou and Chongqing were in 2014 and 2017.

Finally, Chinese HCSE shows a trend first of decline and then rise, reaching a peak in 2017. For the network centrality reflects the equilibrium of network nodes, therefore, HCSE in 2011 and 2017 was relatively concentrated. Combined with the research results of network density, the low network density is in 2011 and 2017 shows that the connections between network members are sparse, making human capital resources concentrated in some areas that leads to an increase in the network centrality; in 2014, the network density was relatively high leading resource and information are able to flow at each node of network, which reduces the network centrality to make HCSE be similar at each node.

\section{The Factors of Spatial Correlation in HCSE Based on the QAP Method}

The previous article analyzed the basic characteristics of the spatial correlation of HCSE, and then this article will specifically explore which factors drive these features.

\subsection{Theoretical Assumptions and Data Selection}

(1) Industrial structure. From the above theoretical basis and analysis results, the spatial correlation of HCSE has various characteristics among the major economic sectors. The eastern coastal areas have obvious spillover effects, while the western regions have an inward siphon effect. In addition, according to some related research, spatial correlation is strongly related to neighboring locations, but it is obvious that the central region does not play the intermediary role of its geographic location. Therefore, we believe that the development mode of provinces and cities has a significant impact on spatial correlation, which we make it is represented by industrial structure. As the primary industry has less and less influence on the overall national economy in modern development, this article uses the ratio of the output value of the tertiary industry to the secondary industry to describe industrial structure.

(2) Investment scale. Generally speaking, investment is one of the "troikas" that drive regional development. On the one hand, it reflects the outside world's expectations for regional development, which will be able to better attract the inflow of related human capital; on the other hand, the high scale of investment can also further promote the region's emphasis on education, thereby promoting the accumulation of human capital. Foreign investment not only has the effect of investment, but also brings new technology and manpower to the region. Therefore, this article uses the level of foreign investment to describe investment scale.

(3) Regional openness. The degree of regional development is conducive to the free distribution of production factor system, which through the role of market in the allocation of factors to achieve a virtuous circle of "self-evolution". Due to differences in regional development, trade between different entities is formed. This not only brings about material flows between regions, but also stimulates the spread of relevant information across regions. This is especially important for narrowing the regional development gap. Therefore, this study selects the trade openness of the region to specifically measure the degree of regional economic development.

(4) Urbanization. The level of urbanization reflects the release of rich rural labor in a region, and promotes the spatial flow and regional accumulation of labor. Therefore, this study incorporates the level of urbanization as an influencing factor.

(5) Transportation infrastructure. The level of transportation infrastructure can not only drive total factor productivity, but also reflect the level of regional development. At the same time, many existing studies have shown that urban commuting can greatly affect the location choice of labor. Therefore, the spatial distribution of human capital is related to the level of transportation infrastructure to a certain extent. In this study, an area's road and railway mileage is used to reflect transportation infrastructure.

So far, this research has established the following model:

$$
\text { HCSU }=f(\text { Ind, Inv, Open, Urban, Infra })
$$


Among them, HCSU represents the matrix of human capital development, Ind, Inv, Open, Urban, Infra represent industrial structure level difference matrix, foreign investment ratio difference matrix, openness level difference matrix, urbanization level difference matrix, transportation infrastructure level difference matrix.

\subsection{Analysis Method and Result Analysis}

From the above-established equation, it can be seen that all variables exist in the form of a relational matrix. Therefore, there may be a high degree of correlation between variables when the data set is established. Therefore, using traditional measurement methods to discuss the relationship between independent variables and dependent variables is more prone to multicollinearity, which makes the final calculation result lose statistical significance. The advantage of QAP is that the non-parametric method is used when testing the causal relationship between variables, and there is no need to assume that the independent variables are independent of each other, which can effectively avoid variables related problems.

\section{(1) QAP correlation analysis}

QAP correlation analysis is mainly based on matrix replacement. It requires the matrix to be a square matrix, so as to compare the similarity of very elements value of the two square matrices to give the overall correlation coefficient, and then do a non-parametric test on the correlation coefficient. The calculation principle is mainly to compare the correlation coefficient of the transformed matrix with the original matrix through continuous and random matrix conversion, then investigate whether the distribution of the correlation coefficient falls into the rejection domain or the acceptance domain. The specific calculation steps will not be repeated. The following table is the calculation result of selecting 5000 random permutation matrixes.

The actual correlation coefficient in Table 5 is directly calculated based on the values of two matrices, the average of the correlation coefficient is calculated based on 5000 random matrix replacements, and the max and min values are the maximum and minimum values of the correlation coefficient in this process. The results show that the spatial correlation between HCSE and the investment scale difference, urbanization difference, and regional openness difference have significant correlation coefficients, which comprehensively indicates that investment scale difference, urbanization development difference, and regional openness difference have a cross-regional relationship with human capital. On the contrary, the industrial structure difference and transportation infrastructure difference have not passed the significance test, indicating that these two types of factors do not directly affect the spatial correlation of HCSE, and may play an intermediary role through other production factors. Therefore, in the follow-up research, these two types of factors are eliminated.

Table 5. Correlation result.

\begin{tabular}{cccccccc}
\hline & Correlation Value & Sig & Average & Min & Max & $\boldsymbol{p} \geq \mathbf{0}$ & $\boldsymbol{p} \leq \mathbf{0}$ \\
\hline Ind & 0.0132 & 0.2705 & 0.0239 & -0.0705 & 0.0891 & 0.2705 & 0.7297 \\
Inv & -0.0541 & 0.0408 & 0.0327 & -0.1056 & 0.1313 & 0.9594 & 0.0408 \\
Urban & -0.001 & 0.4755 & 0.0093 & -0.025 & 0.0399 & 0.5247 & 0.0476 \\
Infra & 0.0029 & 0.4515 & 0.0365 & -0.1683 & 0.1997 & 0.4515 & 0.5487 \\
Open & -0.0541 & 0.0452 & 0.0328 & -0.0934 & 0.1668 & 0.955 & 0.0452 \\
\hline
\end{tabular}

(2)

QAP regression analysis

Table 6 shows the correlation coefficients of QAP regression analysis and the corresponding test indicators. Among them, the probabilities as large and as small respectively represent the probability that the regression coefficient is greater than or equal to and less than or equal to the final regression coefficient during the random replacement process. 
Table 6. Regression result.

\begin{tabular}{ccccccc}
\hline & Unstdized Coef & Stdized Coef & $p$-Value & As Large & As Small & Std Err \\
\hline Urban & 0.03419 & 0.03716 & 0.08038 & 0.08038 & 0.91982 & 0.02561 \\
Open & -0.00084 & -0.01688 & 0.03213 & 0.66807 & 0.03213 & 0.00216 \\
Inv & -0.00427 & -0.06558 & 0.05059 & 0.94961 & 0.05059 & 0.0027 \\
Intercept & -0.00424 & 0 & 0 & 0 & 0 & 0 \\
\hline
\end{tabular}

As can be seen from the results in the table, first, the significance level of each variable passes the test; the difference in urbanization is significant at $10 \%$, and the difference in openness and investment scale are both significant at $5 \%$; second, in terms of influence, compared with urbanization development difference and regional openness difference, foreign investment difference has the greatest effect on the spatial correlation of HCSE in China, followed by the level of urbanization development difference, and finally the degree of regional openness difference; finally, specifically, the smaller the difference in regional attractiveness (regional openness difference), the more conducive to the construction of inter-regional networks for human capital development. That is, those regions with similar development prospects can better unify the network attributes of HCSE then it is easy to accelerate the efficiency of network formation and to form regional human capital accumulation; similarly, the small difference in the degree of regional openness helps to reduce the regional barriers to HCSE, but this will have two different results-either stand still or share mutual assistance. On the contrary, the greater the difference in urbanization level is, the more favorable the regional spillover effect of HCSE is. The main reason is that urbanization can release the surplus labor force in countryside and drive this labor force to move to city. Through continuous learning and retraining, their human capital is effectively improved. When this part of the labor force returned, they brought new knowledge and technology to their original hometown. Such a continuous cycle promotes the establishment of a regional human capital network and HCSE. The relevant research on the reemployment of Chinese migrant workers in their hometowns just proved the above conclusions.

\section{Research Conclusions and Policy Recommendations}

\subsection{Research Conclusions}

On the basis of a continuous flow of production factors among regions, enhancing the level of human capital to realize regional high-level human capital structure and its spatial aggregation and spillover effect with the result of making the human capital structure and economic structure formed between dynamic collaborative development, it is the way to ensure that China's overall economic "soft landing" to realize the economic sustainable development, and it is also an important means to build a well-off society in an all-round way as well as the key to reduce regional development potential difference. Therefore, a comprehensive and objective assessment of the degree of human capital structure upgrading and its characteristics of spatial network structure in China as a whole and in various provinces will help clarify the current situation of human capital development to provide a strong support and reference basis for formulating human capital policies that are more in line with the development of localities. Previous studies have mostly been based on the stock of human capital, lacking in consideration of the spatial network structure formed by the flow of human capital. Based on the data of Chinese provinces and cities from 2008 to 2018, combined with the homogeneity and heterogeneity of human capital and the modified spatial matrix, we construct the evolution model of human capital structure of various provinces and cities in China. Then by using social network analysis method, the spatial correlation and main influencing factors are empirically investigated. The main findings are as follows:

(1) HCSE in various provinces and cities was relatively small except for the large fluctuations in 2009-2012 and 2014-2015. From a formal point of view, HCSE in the eastern region relies mainly on external spillover effects, while the central and western regions have both external output and internal input. At the same time, the correlation dimension 
of phase space reconstruction has fluctuated greatly in 2008 2018. In 2008 2011, 2013 2014, 2016 2017, HCSE in China was relatively balanced, while in other years, the characteristics of regional agglomeration appeared.

(2) From the perspective of overall network characteristics, during the investigation period, the spatial network density of HCSE showed a trend of increasing first and then decreasing, indicating that the inter-provincial human capital connection is not stable, but only in 2014 the connection effect was the best. The research results of the small world network analysis show that the agglomeration effect is gradually increasing, and the average distance of correlation is relatively close, so it has better spatial correlation and spatial spillover effects.

(3) From the perspective of the characteristics of individual network structure, the eastern region (Beijing, Shanghai, and Guangdong are important members) has higher degree centrality, proximity centrality, and intermediary centrality, the central region (Hunan and Henan is the main province) only had high proximity centrality in 2014, the western region (mainly Guizhou, Chongqing and Shaanxi) had a high degree of centrality and intermediary centrality in 2017.We can understand that the eastern region has a strong central position in network, and can also form a good spillover effect through its human capital accumulation. Although the western region has the advantage of being close to resources and intermediary power in the network structure in some individual years based on introducing human capital, so as long as its existing path dependence is enhanced, then it cannot generate high-level human capital internally; while the central region is more modest.

(4) The scale of investment, the level of urbanization, and the degree of urban openness are the main factors that produce the spatial correlation of HCSE. Using the QAP correlation analysis conducted by the QAP secondary assignment procedure, this study found that the differences in urbanization levels between provinces and municipalities have a positive impact on spatial correlation, while differences in investment scale and urban openness have significant negative effects.

\subsection{Policy Recommendations}

(1) Comprehensively understanding the stock, dynamic changes and spatial mobility of human capital means shifting from single linear thinking that focuses on the stock of human capital to promoting human capital structures, upgrading them to promote further sustained and rapid growth of innovation. The concept of HCSE comes from a full understanding of the stock of human capital, the homogeneity and heterogeneity of human capital, and its dynamic changes. Combining our research results, we believe that in the face of the differences in HCSE between provinces in China, local governments should gradually promote the transformation of human capital structures according to their development characteristics and development needs, so as to ultimately drive the internal upgrading of human capital structure. In addition, given the different regional development history, it is easy to form a relatively fixed development model and corresponding people, namely the path dependence is obvious. Therefore, governments need to plan in-depth the synergy between the successor industry and the local industry to promote HCSE.

(2) Continuously adjust and optimize the spatial correlation network structure on which the advanced path of the human capital structure depends, improve the spatial allocation efficiency of human capital, and realize the coordinated development of space. Although human capital flow has an obvious geographical proximity relationship, which creates favorable conditions for the cross-regional human capital exchange in geographically adjacent areas and lowers the flow barriers. Due to the complexity of economic activities, we cannot fully explain the characteristics of Chinese inter-provincial flow of human capital from the perspective of geographic proximity. The spatial network structure of HCSE provides new impetus for the realization of the cross-regional coordinated development mechanism. Therefore, governments need to pay attention to the geographical "close 
neighbors" as well as re-examine and construct a cross-regional coordinated development strategy from a national macro perspective.

(3) Further individual network structures play in a role of the fairness of HCSE and eliminating the dependence of negative human capital development paths. Some results of this research show that the path dependence of HCSE in some regions plays an important role in the overall network structure, but it includes both positive spillover effect regions and conservative regions as well as negative external dependence regions. So it is necessary for governments to actively explore effective ways to promote the spatial correlation of HCSE, further exert the spillover effects of some important regions, and pay special attention to guiding the negative development regions from the one-way input link to the two-way convection equal exchange, so as to continue narrow the development gap between provinces in the spatial correlation network.

Author Contributions: The initial idea for the paper came from X.D. and she did the main job including Literature collection, the research design, data collection, calculated the results and writing the article. L.Y. gave a lot of support to guide discussion and revision. Both authors have read and agreed to the published version of the manuscript.

Funding: The National Natural Science Foundation of China (Grant No. 71273246); The crucial project of philosophical and social science study of the ministry of education in China (Grant No. 12JZD034).

Institutional Review Board Statement: Not applicable.

Informed Consent Statement: Informed consent was obtained from all subjects involved in the study. Data Availability Statement: The data I used are shown in the article.

Acknowledgments: The authors gratefully acknowledge the financial support provided by the National Natural Science Foundation of China (Grant No. 71273246) and the crucial project of philosophical and social science study of the ministry of education in China (Grant No. 12JZD034).

Conflicts of Interest: The authors declare no conflict of interest.

\section{Appendix A}

Table A1. Network Centrality.

\begin{tabular}{|c|c|c|c|c|c|c|c|c|c|}
\hline \multirow{2}{*}{ Time } & \multicolumn{3}{|c|}{2011} & \multicolumn{3}{|c|}{2014} & \multicolumn{3}{|c|}{2017} \\
\hline & Degree & Closeness & Betweenness & Degree & Closeness & Betweenness & Degree & Closeness & Betweenness \\
\hline Beijing & 62.963 & 72.973 & 8.926 & 40.741 & 60.000 & 1.314 & 48.148 & 65.854 & 2.198 \\
\hline Hebei & 48.148 & 64.286 & 3.630 & 37.037 & 58.696 & 1.124 & 51.852 & 67.500 & 2.467 \\
\hline Shanxi & 40.741 & 62.791 & 0.673 & 48.148 & 62.791 & 1.417 & 40.741 & 62.791 & 1.627 \\
\hline Neimenggu & 37.037 & 60.000 & 0.440 & 48.148 & 64.286 & 0.588 & 51.852 & 67.500 & 2.467 \\
\hline Liaoning & 48.148 & 64.286 & 5.953 & 48.148 & 62.791 & 1.417 & 51.852 & 62.791 & 0.399 \\
\hline Jilin & 51.852 & 67.500 & 2.139 & 59.259 & 71.053 & 3.749 & 48.148 & 65.854 & 2.085 \\
\hline Heilongjiang & 44.444 & 64.286 & 1.756 & 55.556 & 69.231 & 4.891 & 48.148 & 65.854 & 2.467 \\
\hline Shanghai & 44.444 & 64.286 & 1.756 & 55.556 & 69.231 & 4.108 & 55.556 & 69.231 & 4.002 \\
\hline Jiangsu & 40.741 & 60.000 & 0.648 & 48.148 & 65.854 & 2.670 & 48.148 & 65.854 & 2.467 \\
\hline Zhejiang & 40.741 & 58.696 & 1.426 & 51.852 & 67.500 & 2.393 & 55.556 & 64.286 & 0.721 \\
\hline Anhui & 40.741 & 60.000 & 0.648 & 55.556 & 69.231 & 3.725 & 40.741 & 62.791 & 1.722 \\
\hline Fuzhou & 37.037 & 61.364 & 2.127 & 51.852 & 67.500 & 2.393 & 29.630 & 50.000 & 0.045 \\
\hline Jiangxi & 55.556 & 65.854 & 3.618 & 48.148 & 65.854 & 0.996 & 33.333 & 51.923 & 0.087 \\
\hline Shandong & 48.148 & 64.286 & 0.959 & 48.148 & 65.854 & 2.670 & 59.259 & 64.286 & 1.571 \\
\hline Henan & 55.556 & 67.500 & 4.355 & 55.556 & 69.231 & 3.042 & 51.852 & 62.791 & 0.399 \\
\hline Hubei & 44.444 & 62.791 & 0.639 & 48.148 & 65.854 & 0.532 & 55.556 & 64.286 & 0.678 \\
\hline Hunan & 48.148 & 64.286 & 0.959 & 48.148 & 65.854 & 0.776 & 66.667 & 75.000 & 4.124 \\
\hline Guangdong & 55.556 & 65.854 & 3.618 & 48.148 & 65.854 & 0.996 & 62.963 & 71.053 & 4.184 \\
\hline Guangxi & 51.852 & 65.854 & 1.823 & 51.852 & 65.854 & 0.739 & 33.333 & 51.923 & 0.087 \\
\hline Chongqing & 55.556 & 69.231 & 1.968 & 51.852 & 67.500 & 1.078 & 62.963 & 67.500 & 1.594 \\
\hline Sichuan & 44.444 & 62.791 & 0.639 & 51.852 & 67.500 & 1.491 & 59.259 & 71.053 & 6.909 \\
\hline Guizhou & 51.852 & 65.854 & 5.120 & 59.259 & 71.053 & 5.188 & 59.259 & 69.231 & 4.597 \\
\hline Yunnan & 33.333 & 58.696 & 1.070 & 55.556 & 69.231 & 1.512 & 59.259 & 69.231 & 4.597 \\
\hline Shanxi & 40.741 & 62.791 & 1.760 & 51.852 & 65.854 & 0.739 & 62.963 & 72.973 & 4.643 \\
\hline Gansu & 44.444 & 62.791 & 0.639 & 51.852 & 65.854 & 0.739 & 59.259 & 65.854 & 1.000 \\
\hline Qinghai & 48.148 & 65.854 & 0.821 & 44.444 & 58.696 & 0.456 & 33.333 & 58.696 & 1.064 \\
\hline Ningxia & 44.444 & 64.286 & 2.058 & 55.556 & 69.231 & 4.852 & 37.037 & 58.696 & 0.567 \\
\hline Xinjiang & 51.852 & 62.791 & 0.799 & 44.444 & 61.364 & 0.248 & 48.148 & 65.854 & 1.631 \\
\hline
\end{tabular}


Table A2. HCSE.

\begin{tabular}{|c|c|c|c|c|c|c|c|c|c|c|c|}
\hline & 2008 & 2009 & 2010 & 2011 & 2012 & 2013 & 2014 & 2015 & 2016 & 2017 & 2018 \\
\hline Anhui & -0.10314 & 0.041671 & 0.033885 & -0.05223 & 0.090433 & 0.041554 & -0.09281 & 0.079402 & -0.17786 & -0.2937 & -0.1864 \\
\hline Beijing & -0.05745 & -0.06352 & -0.07079 & 0.168197 & 0.09959 & 0.101209 & -0.12836 & 0.119859 & -0.01297 & -0.0155 & -0.158 \\
\hline Chongqing & 0.035769 & -0.04286 & -0.03346 & -0.1175 & -0.10839 & -0.02636 & -0.07164 & 0.041574 & 0.175387 & 0.28 & 0.39 \\
\hline Fujian & -0.03846 & 0.023325 & 0.018798 & 0.22078 & -0.07518 & 0.031656 & -0.04564 & 0.037862 & -0.06874 & -0.11 & -0.04 \\
\hline Gansu & 0.02157 & -0.11262 & -0.02918 & 0.17788 & -0.00909 & -0.11021 & 0.011911 & -0.0366 & 0.047578 & 0.09 & 0.074 \\
\hline Guangdong & -0.04063 & -0.08216 & -0.06708 & 0.091888 & -0.16109 & -0.07233 & -0.06319 & 0.100096 & 0.166957 & 0.27 & 0.42 \\
\hline Guangxi & 0.064491 & -0.00748 & 0.006124 & -0.06319 & 0.09994 & -0.03185 & 0.022813 & 0.021551 & -0.11119 & -0.195 & -0.18 \\
\hline Guizhou & 0.012279 & 0.033203 & 0.079224 & -0.13108 & 0.114611 & 0.081989 & 0.07702 & -0.10578 & -0.08491 & -0.13 & -0.3 \\
\hline Hebei & -0.06513 & 0.003977 & -0.04354 & -0.00577 & -0.1524 & 0.076768 & -0.13177 & 0.102466 & 0.022788 & 0.05 & 0.2 \\
\hline Heilongjiang & 0.06193 & 0.016233 & -0.00172 & -0.01526 & -0.02382 & 0.045704 & -0.06953 & 0.02999 & 0.079626 & 0.14 & 0.2 \\
\hline Henan & -0.0287 & -0.00842 & -0.03581 & -0.10487 & -0.09917 & 0.043409 & -0.02639 & 0.038905 & 0.043571 & 0.06 & 0.1 \\
\hline Hubei & 0.035568 & 0.043647 & -0.04563 & 0.03105 & -0.02548 & 0.02452 & 0.057363 & -0.03783 & 0.023935 & 0.03 & -0.05 \\
\hline Hunan & -0.01592 & -0.0478 & 0.040573 & -0.04601 & 0.003569 & -0.00123 & -0.0233 & -0.01719 & 0.009627 & 0.03 & 0.028 \\
\hline Jiangsu & 0.088992 & -0.05231 & -0.09546 & -0.03267 & -0.17485 & -0.0068 & 0.061506 & -0.08705 & 0.279469 & 0.46 & 0.34 \\
\hline Jiangxi & 0.009027 & 0.03022 & 0.052942 & 0.05697 & 0.093962 & -0.03995 & -0.02308 & -0.00708 & -0.09084 & -0.14 & -0.1159 \\
\hline Jilin & -0.04091 & 0.004324 & -0.01952 & -0.0107 & -0.00091 & -0.07558 & 0.060276 & -0.00723 & -0.12086 & -0.215 & -0.248 \\
\hline Liaoning & -0.02472 & -0.02596 & 0.02458 & 0.028934 & 0.02768 & 0.03257 & 0.009244 & -0.01949 & 0.034212 & 0.06 & 0.027 \\
\hline Neimenggu & 0.000576 & 0.031723 & 0.016289 & 0.035859 & 0.084552 & 0.013584 & -0.01101 & -0.04755 & 0.018259 & 0.051 & 0.01 \\
\hline Ningxia & 0.077143 & 0.208497 & 0.001883 & -0.02155 & 0.069067 & -0.04514 & -0.00812 & -0.07665 & 0.048779 & 0.111 & 0.043 \\
\hline Qinghai & -0.0587 & 0.050806 & 0.033302 & -0.14988 & 0.054806 & 0.127101 & -0.02218 & 0.075744 & -0.07972 & -0.16 & -0.11 \\
\hline Shandong & 0.042618 & -0.05245 & 0.029087 & -0.05481 & -0.0305 & -0.08009 & 0.119014 & -0.06034 & 0.104105 & 0.1543 & 0.047 \\
\hline Shanghai & -0.04569 & -0.01341 & 0.032017 & -0.15868 & 0.017101 & 0.051786 & 0.070509 & 0.154599 & -0.04343 & -0.147 & -0.06242 \\
\hline Shanxi & -0.02612 & 0.000983 & 0.06796 & 0.105319 & 0.129441 & -0.00908 & 0.046599 & -0.04486 & -0.09991 & -0.16 & -0.2263 \\
\hline Shanxi & -0.01934 & -0.05198 & -0.02056 & 0.012127 & 0.060873 & -0.03121 & 0.012863 & -0.0329 & -0.04641 & -0.067 & -0.09757 \\
\hline Sichuan & -0.02669 & -0.02369 & -0.01692 & 0.11341 & 0.035711 & -0.02722 & 0.014422 & -0.00245 & -0.09435 & -0.16 & -0.16 \\
\hline Xinjiang & 0.130937 & -0.00053 & 0.054388 & -0.00758 & -0.00423 & -0.03823 & 0.033134 & 0.02975 & -0.03604 & -0.07995 & -0.06438 \\
\hline Yunnan & -0.00303 & 0.112806 & -0.00102 & 0.072547 & 0.029969 & 0.075361 & 0.034359 & -0.05171 & -0.08369 & -0.1381 & -0.22 \\
\hline Zhejiang & -0.00387 & -0.03574 & -0.04658 & 0.037712 & 0.006401 & -0.07791 & -0.03912 & -0.13405 & 0.100524 & 0.2297 & 0.1448 \\
\hline
\end{tabular}

\section{References}

1. Zheng, S.; Du, R. How does urban agglomeration integration promote entrepreneurship in China? Evidence from regional human capital spillovers and market integration. Cities 2020, 97, 102529. [CrossRef]

2. Shen, J.; Chen, C.; Yang, M.; Zhang, K. City Size, Population Concentration and Productivity: Evidence from China. China World Econ. 2019, 27, 110-131. [CrossRef]

3. Thomas, V.; Wang, Y.; Fan, X. Measuring Education Inequality: Gini Coefficients of Education; Social Science Electronic Publishing, Inc.: New York, NY, USA, 2001; Volume 1, pp. 43-50.

4. Nelson, R.R.; Phelps, E.S. Investment in Humans, Technological Diffusion, and Economic Growth. Am. Econ. Rev. 1966, 56, 69-75.

5. Glaeser, E.L.; Ponzetto GA, M.; Tobio, K. Cities, Skills, and Regional Change. Reg. Stud. 2014, 48, 7-43. [CrossRef]

6. Chang, C.F.; Wang, P.; Liu, J.T. Knowledge spillovers, human capital and productivity. J. Macroecon. 2015, 47, 214-232. [CrossRef]

7. Che, Y.; Zhang, L. Human Capital, Technology Adoption and Firm Performance: Impacts of China's Higher Education Expansion in the Late 1990s. Econ. J. 2018, 128, 2282-2320. [CrossRef]

8. Graham, J.W.; Webb, R.H. Stocks and Depreciation of Human Capital: New Evidence from a Present-Value Perspective. Rev. Income Wealth 2010, 25, 209-224. [CrossRef]

9. Guastella, G.; Timpano, F. Knowledge, innovation, agglomeration and regional convergence in the EU: Motivating place-based regional intervention. Rev. Reg. Res. 2016, 36, 121-143. [CrossRef]

10. Arrow, K.J. The Economic Implications of Learning by Doing. Rev. Econ. Stud. 1962, 29, 155-173. [CrossRef]

11. Romer, P.M. Increasing Returns and Long-Run Growth. J. Political Econ. 1986, 94, 1002-1037. [CrossRef]

12. Rober, L. On the mechanics of economic development. J. Monet. Econ. 1999, 22, 3-42.

13. Liu, Z.Y.; Li, H.Z.; Hu, Y.Y.; Li, C. Human Capital Structure Upgrading and Economic Growth: A Reconsideration of Disparities among China's. Econ. Res. J. 2018, 53, 50-63.

14. Luo, Y.; Wang, Y.; Fan, Z.J. Heterogeneous Human Capital, Regional Specialization and Income Disparity-From the Perspective of New Economic Geography. China Ind. Econ. 2013, 2, 31-43.

15. Raul, R.; Jordi, S.; Manuel, A. Regional Economic Growth and Human Capital: The Role Overeducating. Izad Discussion Paper. 2009. Reg. Stud. 2012, 46, 1389-1400.

16. Huggett, M.; Kaplan, G. How Large is the Stock Component of Human Capital? Rev. Econ. Dyn. 2016, 22, 21-51. [CrossRef]

17. Yang, J.; Li, X.S. Education Inequality, Human Capital and Economic Growth: An Empirical Study on China. J. Quant. Tech. Econ. 2007, 24, 37-45.

18. Bennett, D.L. Educational Inequailty in the United States: Methodology and Historical Estimates of Education Gini Coefficients. SSRN Electron. J. 2012. [CrossRef]

19. Jorgenson, D.; Fraumeni, B.M. The Accumulation of Human and Nonhuman Capital, Revisited. Rev. Income Wealth 2017, 63, 227-286.

20. Zhao, W.; Li, F. Heterogeneous labor mobility and regional income gap: An extended analysis of new economic geography model. Chin. J. Popul. Sci. 2007, 21, 27-35. 
21. Hou, C.L.; Yang, X.M.; Lu, J. Spillover Effect of Human Capital Differences in Regional Economic Growth: An Empirical Analysis Base. J. Qingdao Univ. (Nat. Sci. Ed.) 2015, 28, 68-72.

22. Lange, F.; Topel, R. The Social Value of Education and Human Capital. In Handbook of the Economic Education; Elsevier: Amsterdam, The Netherlands, 2006; Volume 1, pp. 459-509.

23. Faigen, B. The Returns to Education: An Empirical Study of Urban China, 1988-2007. Master's Thesis, Lund University, Lund, Sweden, 2012.

24. Dlouhy, K.; Biemann, T. Path dependence in occupational careers: Understanding occupational mobility development throughout individuals' careers. J. Vocat. Behav. 2018, 104, 86-97. [CrossRef]

25. Arthur, W.B. Competing Technologies, Increasing Returns, and Lock-In by Historical Events. Econ. J. 1989, 99, 116-131. [CrossRef]

26. David, P.A. Why are institutions the "carriers of history"? Path dependence and the evolution of conventions, organizations and institutions. Struct. Chang. Econ. Dyn. 1994, 5, 205-220. [CrossRef]

27. Martin, R.R. Lecture in Economic Geography Rethinking Regional Path Dependence: Beyond Lock-in to Evolution. Econ. Geogr. 2010, 86, 1-27. [CrossRef]

28. Lin, Y.F. New Structural Economics: A Theoretical Framework for Rethinking Development and Policy; World Bank: Washington, DC, USA, 2012.

29. Crane, B.; Albrecht, C.; Duffin, K.M.; Albrecht, C. China's special economic zones: An analysis of policy to reduce regional disparities. Reg. Stud. Reg. Sci. 2018, 5, 98-107. [CrossRef]

30. Zhou, M.; Huang, H. The evolution and structural decomposition of regional economic disparity in China:1990 2009. Stat. Decis. Mak. J. 2012, 16, 122-125.

31. Zhang, W.W.; Qi, L. Geographic Concentration of Labor, Industrial Geography and Regional Disparities. China Econ. Q. 2011, 10, 691-708.

32. Liu, B.Z.; Wu, P.; Liu, Y.H. Transportation Infrastructure and the Increase in TFP in China-Spatial Econometric Analysis on Provincial Panel Data. China Ind. Econ. 2010, 3, 54-64.

33. Zhang, S.L.; Li, Y.; Jiu, E.K. Path dependence, market entry and transformation of resources-based city. Econ. Theory Bus. Manag. 2016, 36, 14-27.

34. Xing, C.; Jia, S.; Li, S. Regional Distribution of the Return to Education for Ruralto-Urban Migrants and Its Impact on Migration. Econ. Res. J. 2013, 11, 114-126.

35. Chen, Q.Y.; Qi, L. Technology comparative advantage, labor knowledge spillover and urbanization in transition economies. Manag. World 2014, 11, 47-59.

36. Glaeser, E.L.; Mare, D.C. Cities and Skills. J. Labor Econ. 2001, 19, 316-342. [CrossRef]

37. Anderson, F.; Burgess, S.; Lane, J.I. Citie's Matching and Productivity Gains of Agglomeration. J. Urban Econ. 2007, 61, 112-128. [CrossRef]

38. Yongshui, W. Retest of Growth Effect on Human Capita with Models of Panel Factors and Error Patterns. Stat. Res. $2018,6,9$.

39. Xiaoguang, C. Downward Compatibility of Human Capital and its Implication for the Cross-country Income Level Accounting. Econ. Res. 2005, 4, 46-56.

40. Neffke, F.; Hartog, M.; Boschma, R.; Henning, M. Agents of Structural Change: The Role of Firms and Entrepreneurs in Regional Diversification. Econ. Geogr. 2018, 94, 23-48. [CrossRef]

41. Takens, F. Deteeting strange attractors in turbulence. In Dynamical Systems and T-urbulence; Springer: Berlin, Germany, 1981; pp. 366-381.

42. Bin, C.; Guang-hu, L.I.; Jun, T.; Yong, Z.H.; Peng, C.A.; Jian, H.U.; Yun-song, W.U. Research on Chaotic Sequence Autocorrelation by Phase Space Method. J. Univ. Electron. Sci. Technol. China 2010, 39, 859-863.

43. Rosenstein, M.T.; Collins, J.J.; De, L.C. Reconstruction expansion as a geometry-based framework for choosing proper delay times. Phys. D Nonlinear Phenom. 1994, 73, 82-98. [CrossRef]

44. Mauldin, R.D.; Williams, S.C. Hausdorff dimension in graph directed constructions. Trans. Am. Math. Soc. 1988, 309, 811-829. [CrossRef]

45. Anliang, W.; Chunxin, Y. Grassberger-Procaccia algorithm for evaluating the fractal characteristic of strange attractors. Acta Phys. Sin. 2002, 51, 2719-2729.

46. Wasserman, S.; Faust, K. Social Network Analysis Methods and Applications. Contemporary Sociology; Cambridge University Press: Cambridge, UK, 1994.

47. Borgatti, S.P.; Mehra, A.; Brass, D.J.; Labianca, G. Network analysis in the social sciences. Science 2009, 323, 892-895. [CrossRef] [PubMed]

48. Bernard, H.R. The Development of Social Network Analysis: A Study in the Sociology of Science, Linton C. Freeman. Empirical Press, Vancouver, BC (2004). Soc. Netw. 2005, 27, 377-384. [CrossRef]

49. Scott, J.; Carrington, P.J. The SAGE handbook of social network analysis. In The SAGE Handbook of Social Network Analysis; SAGE: Thousand Oaks, CA, USA, 2011.

50. Watts, D.J.; Strogatz, S.H. Collective Dynamics of Small World Networks. Nature 1998, 393, 440-442. [CrossRef] [PubMed] 